\title{
ISO mid-infrared spectroscopy of Galactic Bulge AGB stars ${ }^{\star \star \star}$
}

\author{
J. A. D. L. Blommaert ${ }^{1}$, M. A. T. Groenewegen ${ }^{1}$, K. Okumura ${ }^{2}$, S. Ganesh ${ }^{3}$, A. Omont ${ }^{4}$, J. Cami ${ }^{5}$, I. S. Glass ${ }^{6}$, \\ H. J. Habing ${ }^{7}$, M. Schultheis ${ }^{8}$, G. Simon ${ }^{9}$, and J. Th. van Loon $^{10}$ \\ 1 Inst. voor Sterrenkunde, K.U.Leuven, Celestijnenlaan 200 B, 3001 Leuven, Belgium \\ e-mail: jorisb@ster.kuleuven.be \\ 2 Service d'Astrophysique, CEA/DAPNIA, Saclay, France \\ 3 Physical Research Laboratory, Ahmedabad, India \\ 4 Institut d'Astrophysique de Paris, CNRS, Paris, France \\ 5 SETI Institute, NASA Ames Research Center, USA \\ 6 South African Astronomical Observatory, South Africa \\ 7 Leiden Observatory, Leiden, The Netherlands \\ 8 CNRS UMR6091, Observatoire de Besançon, Besançon, France \\ 9 GEPI, Observatoire de Paris, France \\ 10 Astrophysics Group, School of Physical \& Geographical Sciences, Keele University, UK
}

Received 31 July 2006 / Accepted 6 September 2006

ABSTRACT

\begin{abstract}
Aims. To study the nature of Bulge AGB stars and in particular their circumstellar dust, we have analysed mid-infrared spectra obtained with the ISOCAM CVF spectrometer in three Bulge fields.

Methods. The ISOCAM 5-16.5 $\mu \mathrm{m}$ CVF spectra were obtained as part of the ISOGAL infrared survey of the inner Galaxy. A classification of the shape of the $10 \mu \mathrm{m}$ dust feature was made for each case. The spectra of the individual sources were modelled using a radiative transfer model. Different combinations of amorphous silicates and aluminium-oxide dust were used in the modelling. Results. Spectra were obtained for 29 sources of which 26 are likely to be Bulge AGB stars. Our modelling shows that the stars suffer mass loss rates in the range of $10^{-8}-5 \times 10^{-7} M_{\odot} / \mathrm{yr}$, which is at the low end of the mass-loss rates experienced on the thermally pulsing AGB. The luminosities range from 1700 to $7700 L_{\odot}$ as expected for a population of AGB stars with $M_{\text {init }}$ of $1.5-2 M_{\odot}$. In agreement with the condensation sequence scenario, we find that the dust content is dominated by $\mathrm{Al}_{2} \mathrm{O}_{3}$ grains in this sample of low mass-loss rate stars.
\end{abstract}

Key words. stars: AGB and post-AGB - stars: evolution - stars: circumstellar matter - Galaxy: bulge - infrared: stars

\section{Introduction}

Asymptotic Giant Branch (AGB) stars undergo substantial mass loss $\left(10^{-8}-10^{-4} M_{\odot} \mathrm{yr}^{-1}\right)$ which effectively drives their stellar evolution (e.g. Habing 1996). Radial pulsations lift matter high above the photosphere where it cools down and a complex chemistry - based on either carbon or oxygen preponderance in the photosphere - is initiated, leading to the formation of molecules and dust. Radiation pressure from the central star blows away the dust particles, which in turn collide with the gas causing the massive outflow.

The formation scenario and the nature of dust in the circumstellar environment of oxygen-rich AGB stars is still very much an issue for debate. On the basis of IRAS LRS data, several studies were performed to investigate the dust of large samples of AGB stars of different variability types and mass-loss rates (e.g. Onaka et al. 1989; Stencel et al. 1990; Little-Marenin et al. 1990; Sloan \& Price 1995, 1998; Hron et al. 1997). Another study, using groundbased $10 \mu \mathrm{m}$ spectroscopy, was performed

* Based on observations with ISO, an ESA project with instruments funded by ESA Member States (especially the PI countries: France, Germany, the Netherlands and the United Kingdom) and with the participation of ISAS and NASA.

$\star \star$ Full Fig. 2 is only available in electronic form at http://www . aanda. org by Speck et al. (2000). In the $10 \mu \mathrm{m}$ region, a sequence of features was found starting from broad ones peaking longward of $11 \mu \mathrm{m}$ and ending with narrow ones peaking at $9.7 \mu \mathrm{m}$. The interpretation of the broad feature is that it is caused by alumina (amorphous $\mathrm{Al}_{2} \mathrm{O}_{3}$ ) whereas that at $9.7 \mu \mathrm{m}$ is the "classic" silicate feature caused by amorphous silicate dust. Between the two extremes several intermediate types exist and different attempts have been made to classify them, based on observational characteristics (Little-Marenin et al. 1989; Sloan \& Price 1995, 1998; Speck et al. 2000).

In the theoretical dust condensation sequence (Tielens 1990) it is expected that refractory oxides will form first, close to the star at a relatively high temperature of about $1500 \mathrm{~K}$. If the densities in the cooler regions further out in the circumstellar shell are high enough, gas-solid and solid-solid reactions will further transform these minerals and lead to the formation at lower temperatures of silicate dust. If at some intermediate step in the dust condensation sequence however the densities are too low for a certain reaction to occur, the condensation stops at that point and may not continue to the formation of silicate dust this is known as "freeze-out" of the dust condensation sequence. Consequently, stars with low mass loss rates will predominantly exhibit an oxide mineralogy - since the densities are too low for any further step to occur - while stars with high mass loss rates will form silicate dust in copious amounts. An alternative 
scenario was suggested by Sloan \& Price (1998) where the $\mathrm{C} / \mathrm{O}$ ratio drives the condensation sequence. According to these authors, stars with low $\mathrm{C} / \mathrm{O}$ ratios produce silicate dust whereas stars with high $\mathrm{C} / \mathrm{O}$ ratios will produce mainly $\mathrm{Al}_{2} \mathrm{O}_{3}$ grains. Since it is generally believed that both mass loss rates and the $\mathrm{C} / \mathrm{O}$ ratio of oxygen-rich AGB stars increase as a star evolves on the AGB, studying the evolution of the circumstellar dust as a star evolves on the AGB provides a way to discriminate between the two scenarios. Indeed, in the first scenario (dust condensation sequence and freeze-out) one expects to see oxide dust in the earlier phases of the AGB when the mass loss rate is still low, and an increasing silicate dust content as the mass loss rate increases with the evolution of the star along the AGB. Such a time evolution is exactly opposite to what should happen according to the scenario suggested by Sloan \& Price (1998). Work done by Heras \& Hony (2005) on ISO-SWS spectra of a Galactic AGB star sample and by Dijkstra et al. (2005) on ISO-PHT and ISO-CAM spectra of AGB stars in the Magellanic Clouds support the sequence where the formation of the different dust types is related to the mass-loss rates.

A serious limitation to the previous studies has been that because of the limited sensitivities of groundbased, IRAS and even of the ISO-SWS instruments, only relatively nearby bright objects have been observed spectroscopically. With ISO-PHT and ISO-CAM it was possible to observe stars in the Magellanic Clouds, but only the brightest ones. The stars in these studies represent a wide range in parameters such as stellar masses, mass-loss rates and variability characteristics. The evolutionary connection between the different stars and the interpretation of the observational sequences in terms of e.g. dust formation scenarios or stellar evolution has proved difficult to establish.

In this paper we present ISOCAM - CVF $(5-16.5 \mu \mathrm{m}) \mathrm{spec}-$ tral measurements on a more homogeneous sample of AGB stars in three "intermediate" galactic bulge fields $\left(|b| \geq 1^{\circ}\right)$ which were observed as part of the ISOGAL survey (Omont et al. 2003). The ISOGAL survey provided a 5-band point source catalogue including near-infrared (DENIS), 7 and $15 \mu \mathrm{m}$ band photometry. In total about 16 square degrees towards the inner Galaxy were observed down to a flux level of 10-20 mJy in the midinfrared, detecting $\approx 10^{5}$ sources, mostly AGB stars, red giants and young stars. The survey has resulted in a complete sample of AGB stars in the galactic bulge fields. Omont et al. (1999) and Ojha et al. (2003, hereafter OOS) demonstrated that the ISOGAL sources in these fields are AGB stars with a range of mass-loss rates between $10^{-8}$ and $10^{-5} M_{\odot} \mathrm{yr}^{-1}$. The stars at these latitudes in the direction of the Bulge are believed to belong to the Bulge population which show only a small range in masses. According to Zoccali et al. (2003), the Bulge giants have evolved from a population of stars of $\approx 1 M_{\odot}$. In their analysis of Bulge Mira variables, Groenewegen \& Blommaert (2005) find slightly higher stellar masses: 1.5 to $2 M_{\odot}$. We do not know the metallicities of our sample of AGB stars. Rich \& Origlia (2005) find for a sample of M giants in the Baade's Window that their metallicity distribution peaks at slightly subsolar metallicity $(\langle[\mathrm{Fe} / \mathrm{H}]\rangle=-0.190 \pm 0.020$ with a $1 \sigma$ dispersion of $0.080 \pm$ $0.015)$. Although the metallicities of the stars in our sample may be uncertain, we will assume that the main difference between individual objects is their age on the AGB, which is characterized by different luminosities and mass-loss rates.

A further description of the sample is given in Sect. 4. In the following sections we describe the ISO observations and the data reduction. After the presentation of the spectra in Sect. 5 we present the results of radiative transfer modelling. These results are discussed in Sect. 7.
Table 1. CAM CVF observations.

\begin{tabular}{lccr}
\hline \hline Field name & $\begin{array}{c}\text { Centre coordinates } \\
\text { J2000 }\end{array}$ & $\begin{array}{c}\text { ISO TDT } \\
\text { number }\end{array}$ & $\begin{array}{r}\text { Observation } \\
\text { date }\end{array}$ \\
\hline C32 & $174127.20-282739.9$ & 83800324 & 02-Mar.-1998 \\
C35 & $174945.84-292034.8$ & 83800427 & 02-Mar.-1998 \\
OGLE & $175514.49-294030.9$ & 84700430 & 11-Mar.-1998 \\
\hline
\end{tabular}

\section{Observations}

Three fields towards the galactic bulge were observed using the ISOCAM Circular Variable Filter (CVF) over the wavelength range of 5 to $16.5 \mu \mathrm{m}$ (Cesarsky et al. 1996; Blommaert et al. 2003). The individual pixels used were $6^{\prime \prime}$ square, resulting in a total field of view of about $3^{\prime} \times 3^{\prime}$. Table 1 gives the coordinates of the central position of the image, the observation date and the ISO Target Dedicated Time (TDT) number for each field. At each step of the "Long-Wavelength" $(5-16.5 \mu \mathrm{m}) \mathrm{CVF}$, six frames of $2.1 \mathrm{~s}$ duration were collected. The observations form part of a larger set of CVF observations performed on ISOGAL fields (Omont et al. 2003). The main goal of those observations was to study extended emission with a spectrum peaking at the longer wavelengths. In order to get the fastest stabilization of the ISOCAM detector the CVF was scanned from long to short wavelengths. In our analysis we also make use of the $J, H$ and $K$ magnitudes from the 2MASS catalogue (Cutri et al. 2003) and the $I, J, K$, [7] and [15] magnitudes taken from the ISOGAL catalogue (Omont et al. 2003). The $I, J$ and $K$ magnitudes of the latter were obtained by the DENIS team in coordination with the ISOGAL project and come from dedicated measurements of the inner Galaxy and can slightly differ from what is available from the DENIS catalogue (Epchtein et al. 1999; Schuller et al. 2003).

The Bulge fields were selected to have homogeneous and relatively low extinction. $\mathrm{C} 32\left(l=0^{\circ}, b=1^{\circ}\right)$ was observed several times in the ISOGAL survey (Omont et al. 1999). C35 is directly opposite in latitude. The so-called OGLE field $\left(l=0.3^{\circ}\right.$, $\left.b=-2.2^{\circ}\right)$ is a subfield of one of the fields observed by the OGLE team (Udalski et al. 2002) in search of micro-lensing events.

All sources for which we were able to obtain spectra are presented in Table 2 together with the photometry taken from the 2MASS, DENIS and ISOGAL surveys. We found ISOGAL counterparts for all sources except for the sources at RA, Dec (J2000) positions 174941.1-291921 and 174946.1-291944 (but which were detected in the DENIS and 2MASS surveys).

\section{Data reduction}

The data reduction and analysis were performed within the CIA package (Ott et al. 1997). Dark current correction was applied by using the so-called "Vilspa" method which takes time- and temperature-dependencies into account. Cosmic ray hits were removed by applying the deglitch $M M$ method. The data cubes, containing the readout images, were averaged per CVF step over the "valid" data points. Different flatfields from the calibration files were tried out on the data until the best correction was found. The ISOCAM data also suffer from transients. After every flux step the detector needs to stabilize to a new signal (Blommaert et al. 2003). This transient-behaviour is generally treated by applying the so-called Fouks-Schubert (FS) method (Coulais \& Abergel 2000). For our data it emerged that the application of FS does not change the shape of the spectrum. Applying FS however causes an increase in the noise and we 
thus decided not to apply it. The main reason why FS makes no difference is likely to come from the fact that the background is so strong that the integration time per CVF step is sufficient to stabilize the detector for the small flux steps that we observe.

Sources were identified in the images and a spectrum was determined by fitting a point spread function to the point source at each CVF step. The Spectral Response Function of the CVF was determined from standard stars by using aperture photometry and then correcting for the part of the PSF outside the aperture (Blommaert et al. 2001). PSF-fitting and aperture photometry give slightly different results for which we applied corrections. The main difficulty with analysing the CVF data is the problem of the parasitic light. Reflections within the instrument cause "ghost" images (Blommaert et al. 2003; Okumura et al. 1998). The strong background observed in our fields of view (especially at 1 degree from the galactic centre, less for the OGLE field) causes an extended structure in the image, which in principle does not hamper a point source's spectrum as it is removed in the background subtraction. However, especially at the edge of this extended structure, the background subtraction may not be adequate. Strong point sources can create fake sources in other parts of the image or cause ghost light to fall on top of another point source. We carefully checked that all sources were genuine stars by comparing with the ISOGAL rasters (which were observed through ISOCAM filters, and do not suffer from this problem). We also verified whether the ghost reflections interfered with genuine spectra. Fortunately, there is a feature of the reflected light that comes to our aid, namely the fact that the ghost light is very different in the overlap region between the two CVF parts at around $9 \mu \mathrm{m}$. If the spectrum showed a clear jump at these edges of the CVF, then it was clear that it was still suffering from parasitic light. This was checked for all our sources. Only one source, J174127.9-282816, showed the discrepancy between the two CVF parts. It may have been caused by a ghost coming from the bright source J174126.6-282702. The spectrum of the affected star is not included in this paper.

\section{Sample description, a comparison with the ISOGAL Bulge survey}

As was described in Sect. 2, the CVF observations were targeted on three Bulge-fields for which also the regular ISOGAL survey rasters were available. The C32 field was first described in Omont et al. (1999). OOS described the results obtained from all "intermediate" Bulge fields $\left(|l|<2^{\circ},|b| \sim 1-4^{\circ}\right)$. In those papers it was demonstrated that basically all point sources detected at the mid-infrared wavelengths are red giant stars.

Most of the ISOGAL $15 \mu \mathrm{m}$ sources and certainly the sources discussed in this paper, have $\left(K_{\mathrm{s}}\right)_{\mathrm{o}}<8.2 \mathrm{mag}$ and are above the RGB limit. A comparison by Glass et al. (1999) of ISOGAL data with a spectroscopic survey of Baade's window NGC 6522, shows that M giants as early as M 2 are detected by ISOGAL but that it is complete from M 5 onwards. Alard et al. (2001, ABC from now on) combined the ISOGAL data with data coming from the gravitational lensing experiment MACHO for the Baade windows. In contrast to earlier photographic searches for variable stars (e.g. Lloyd Evans 1976), the MACHO survey found variability down to much smaller amplitudes (i.e, $0.5 \mathrm{mag}$ could be detected in the older work, compared to better than 0.1 mag in the new). ABC concluded that almost all sources detected at 7 and $15 \mu \mathrm{m}$ and thus above the RGB-limit are variable. Both semi-regular and Mira-type variables are present in the ISOGAL sample, and the first group of variables outnumbers the latter by about 20:1. We have no variability information
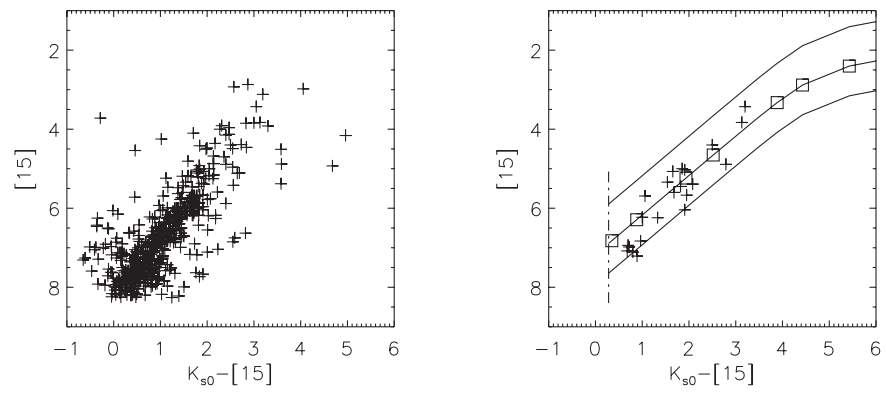

Fig. 1. $[15] /\left(K_{\mathrm{s}}-[15]\right)_{\mathrm{o}}$ colour-magnitude diagrams. The left figure shows all sources detected in our selected ISOGAL fields. A linear sequence of increasingly redder colours (and thus higher mass-loss rates) for brighter $15 \mu \mathrm{m}$ fluxes can be seen. The right box shows the sources observed with the CVF, illustrating the fact that we have a representative sample which covers the sequence of increasing mass-loss rate. For comparison, the right box also shows colors and magnitudes corresponding to theoretical models of mass-losing AGB stars with various luminosities and mass loss rates (see Sect. 6 for more technical details). The dot-dashed line represents a track of increasing luminosity without mass loss. The other three curves represent giants with luminosities of 2000 (bottom), 4000 (middle) and $10000 L_{\odot}$ (top) with increasing mass-loss rate (using a mixture of amorphous silicate and amorphous aluminium oxide dust). The squares on the $4000 L_{\odot}$ track indicate the following mass-loss rates: $10^{-9}, 10^{-8}, 5 \times 10^{-8}, 10^{-7}, 5 \times 10^{-7}, 10^{-6}$, $3 \times 10^{-6} M_{\odot} / \mathrm{yr}$.

for the sources observed with the CVF but expect our sample to be dominated by semi-regular variables.

A [15] vs. $\left(K_{\mathrm{s}}-[15]\right)_{\mathrm{o}}$ diagram of the sources for which we obtained a mid-infrared spectrum is shown in Fig. 1. In comparison with the [15] vs. $\left(K_{\mathrm{s}}-[15]\right)_{\mathrm{o}}$ diagram given in OOS, it shows that we have a good coverage of the range of giant stars detected with ISOGAL. In Omont et al. (1999) and OOS the clear linear sequence of increasing $\left(K_{\mathrm{s}}-[15]\right)_{\mathrm{o}}$ colour for brighter $15 \mu \mathrm{m}$ fluxes is interpreted as a combination of increasing luminosities and increasing mass-loss rates. The reddest colours cannot be explained by very cold photospheres, but are influenced by dust emission. The exact amount of the total mass-loss rate is modeldependent, but is of the order of $10^{-9}$ to a few $10^{-7} M_{\odot} / y r$. Our sample is dominated by stars with relatively low mass-loss rates in comparison to the often studied Miras or even OH/IR stars ( $\dot{M} \geq 10^{-6} M_{\odot} / \mathrm{yr}$ ) but this is simply due to the fact that we have a "blind" survey and that there are many more AGB stars with moderate mass-loss rates (OOS). For comparison we have plotted theoretical tracks with increasing mass loss rates for different luminosities in Fig. 1. The modelling used for these tracks is discussed in Sect. 6.

\section{Presentation of the spectra}

Figure 2 shows the spectra of our sources together with their model fits (see Sect. 6) and the spectra corrected for extinction by using the dereddening law of Cardelli et al. (1989). As this extinction law does not include any interstellar silicate extinction in the 10 micron region, the extinction provided by astronomical silicate (Draine 1985) was added beyond $7 \mu \mathrm{m}$, scaled to give $A_{9.5 \mu \mathrm{m}}=0.077 A_{\mathrm{V}}$. The $A_{\mathrm{v}}$ values (see Table 2) were taken from Schultheis et al. (1999). If no adjacent extinction value was available we used the value obtained per field on basis of DENIS photometry (OOS): $A_{\mathrm{V}}=6.3,8.1$ and 2.2 for C32, C35 and the OGLE field respectively.

The extinction values are sufficiently high, especially in C32 and $\mathrm{C} 35$, to have quite an impact on the spectrum even at 
Table 2. The sources for which spectra were obtained. The magnitudes from the ISOGAL catalogue (DENIS-bands and ISOCAM 7 and $15 \mu \mathrm{m}$ ) are given together with the 2MASS data. The uncertainties for the DENIS magnitudes are typically 0.05 mag and 0.15 mag for the ISO photometry (Schuller et al. 2003). The uncertainties for the 2MASS photometry are given between brackets. The interstellar extinction values are taken from Schultheis et al. (1999). If no extinction value was available for a source, we used the value obtained per field on basis of DENIS photometry (OOS): $A_{\mathrm{V}}=6.3,8.1$ and 2.2 for C32, C35 and the OGLE field respectively. (*: For the 3 sources which we suspect to be foreground, we use a lower $A_{\mathrm{V}}=1.5$ value). For two sources (at positions 174941.1-291921 and 174946.1-291944) we have no ISOGAL counterpart and we have given the position and photometry taken from the 2MASS and DENIS surveys.

\begin{tabular}{|c|c|c|c|c|c|c|c|c|c|}
\hline ISOGAL name & $I_{\text {DENIS }}$ & $J_{\text {DENIS }}$ & $K_{\text {DENIS }}$ & $J_{2 \mathrm{MASS}}$ & $H_{2 \mathrm{MASS}}$ & $K_{2 \mathrm{MASS}}$ & [7] & [15] & $A_{\mathrm{V}}$ \\
\hline \multicolumn{10}{|l|}{ C32 } \\
\hline $\mathrm{J} 174121.4-282810$ & 10.43 & 8.66 & 7.26 & $8.61(0.02)$ & $7.70(0.03)$ & $7.40(0.03)$ & 6.88 & & $1.5^{*}$ \\
\hline $\mathrm{J} 174123.6-282723$ & 16.17 & 10.65 & 8.26 & & $8.99(0.03)$ & $8.25(0.03)$ & 7.57 & 6.98 & 6.13 \\
\hline $\mathrm{J} 174124.7-282801$ & & 10.80 & 8.51 & $10.46(0.03)$ & $8.95(0.03)$ & $8.39(0.03)$ & 6.99 & 6.04 & \\
\hline $\mathrm{J} 174125.7-282807$ & 17.21 & 10.72 & 7.83 & $10.64(0.04)$ & $8.88(0.04)$ & $7.97(0.02)$ & 6.85 & 5.45 & \\
\hline $\mathrm{J} 174126.6-282702$ & & 11.16 & 7.52 & $11.56(0.03)$ & $9.29(0.03)$ & $7.90(0.02)$ & 5.44 & 3.83 & \\
\hline $\mathrm{J} 174127.3-282851$ & 16.54 & 10.26 & 7.46 & $10.01(0.02)$ & $8.30(0.04)$ & $7.30(0.02)$ & 5.78 & 4.40 & \\
\hline $\mathrm{J} 174128.5-282733$ & 15.23 & 10.00 & 7.44 & $9.58(0.02)$ & $7.97(0.04)$ & $7.11(0.02)$ & 6.41 & 5.34 & 6.00 \\
\hline J174130.2-282801 & & 11.35 & 8.74 & $11.35(0.05)$ & $9.66(0.04)$ & $8.88(0.03)$ & 7.77 & 7.44 & \\
\hline $\mathrm{J} 174131.2-282815$ & 14.96 & 9.78 & 7.31 & $9.80(0.03)$ & $8.03(0.03)$ & $7.30(0.03)$ & 6.45 & 5.69 & 6.00 \\
\hline $\mathrm{J} 174133.7-282723$ & 16.47 & 10.93 & 8.32 & $10.80(0.04)$ & $9.12(0.04)$ & $8.38(0.04)$ & 7.49 & 7.08 & \\
\hline J174134.1-282653 & 12.00 & 9.37 & 7.52 & $9.32(0.02)$ & $8.07(0.03)$ & $7.60(0.02)$ & 7.26 & 7.29 & $1.5^{*}$ \\
\hline \multicolumn{10}{|l|}{ C35 } \\
\hline 174941.1-291921 & & 11.72 & 8.93 & $11.65(0.04)$ & $9.71(0.03)$ & $8.82(0.03)$ & & & 8.13 \\
\hline J174942.3-292043 & & 11.44 & 8.01 & $10.91(0.11)$ & & & 6.23 & 4.89 & \\
\hline J174943.3-291947 & 14.84 & 10.45 & 7.20 & $11.33(0.04)$ & $9.02(0.03)$ & $7.71(0.02)$ & 4.94 & 3.43 & 8.00 \\
\hline J174943.7-292154 & 17.55 & 10.61 & 7.83 & $10.81(0.03)$ & $8.89(0.02)$ & $7.97(0.04)$ & 6.97 & 6.23 & 7.75 \\
\hline J174944.5-292009 & 17.66 & 11.35 & 8.23 & $11.26(0.03)$ & $9.22(0.03)$ & $8.14(0.02)$ & 6.94 & 5.67 & 8.00 \\
\hline 174946.1-291944 & 15.95 & 11.03 & 8.36 & $10.99(0.03)$ & $9.08(0.03)$ & $8.16(0.03)$ & & & 7.67 \\
\hline J174946.4-292005 & 16.97 & 11.13 & 8.04 & $11.05(0.03)$ & $9.02(0.03)$ & $8.02(0.02)$ & 6.65 & 5.39 & 7.67 \\
\hline J174946.5-291917 & & 11.34 & 8.63 & $11.46(0.03)$ & $9.53(0.04)$ & $8.68(0.03)$ & 7.83 & & 7.38 \\
\hline J174946.5-291933 & 14.44 & 10.39 & 7.52 & $10.34(0.03)$ & $8.34(0.04)$ & $7.36(0.03)$ & 6.16 & 5.03 & 7.38 \\
\hline J174948.1-292104 & 17.63 & 11.38 & 8.60 & $11.40(0.03)$ & $9.52(0.03)$ & $8.56(0.03)$ & 7.75 & 7.11 & 6.50 \\
\hline $9.2-291932$ & 17.23 & 10.55 & 7.79 & $10.57(0.03)$ & $8.72(0.03)$ & $7.83(0.02)$ & 6.84 & 5.60 & \\
\hline J174951.7-292108 & 14.59 & 10.90 & 8.15 & $10.83(0.03)$ & $9.04(0.03)$ & $8.12(0.03)$ & 7.39 & 6.24 & 6.88 \\
\hline \multicolumn{10}{|l|}{$O G L E$} \\
\hline J175511.9-294027 & 12.83 & 10.30 & 8.77 & $10.42(0.05)$ & $9.25(0$. & 8.84 & 8.17 & 7.89 & \\
\hline 4.1-293928 & 12.25 & 9.54 & 7.99 & $9.54(0.03)$ & $8.42(0.02)$ & 8.00 & 7.22 & 6.83 & \\
\hline 4.9-293918 & 11.04 & 8.75 & 7.16 & - & - & $7.11(0.03)$ & 6.73 & 6.42 & $1.5^{*}$ \\
\hline 5.3-294016 & 13.98 & 9.25 & 7.24 & $9.10(0.04)$ & $7.85(0.03)$ & $7.18(0.02)$ & 6.06 & 5.00 & \\
\hline J175518.9-294142 & 13.57 & 8.88 & 7.07 & $8.85(0.03)$ & $7.62(0.03)$ & $7.02(0.02)$ & 6.28 & 5.07 & \\
\hline J175521.0-294055 & 12.28 & 9.77 & 8.20 & $9.77(0.03)$ & $8.68(0.02)$ & $8.27(0.03)$ & 7.85 & 7.76 & \\
\hline
\end{tabular}

mid-infrared wavelengths. An example of this is shown in Fig. 3. Because of the strong silicate feature in the interstellar environment, it depresses the silicate emission in the observed spectrum and gives the impression that the mid-infrared spectrum peaks at wavelengths beyond ten microns.

As suggested from the ISOGAL work (see previous section), our spectra are indeed typical for mass-losing AGB stars: still dominated by the photosphere below $9 \mu \mathrm{m}$, showing different molecular absorption bands and with dust features clearly apparent at longer wavelengths. We will discuss these dust features in the next subsection.

\subsection{The $10 \mu m$-feature}

Almost all spectra show a clear mid-infrared excess over the photospheric emission. A strong $10 \mu \mathrm{m}$-feature is apparent, but with a considerable variation in shape. Several papers discuss the different shapes and origins of what is often called for convenience the "silicate" feature.

Sloan \& Price (1995, hereafter SP) describe a sequence of $10 \mu \mathrm{m}$ emission features. The sequence starts with a broad, low contrast feature, peaking longward of $11 \mu \mathrm{m}$. At the other extreme of the sequence one finds the narrow silicate emission feature that peaks at $10 \mu \mathrm{m}$. SP subdivide the sequence to classify the different shapes. As their method is quantitative, we apply it to our spectra. To isolate the dust feature we need to subtract the photospheric emission. In the SP analysis, this is done by using an Engelke function (which is a modified Planck function, Engelke 1992) at $3240 \mathrm{~K}$ corresponding to the effective temperature of an M6 giant, which was the average spectral type of their sample. On the basis of Glass et al. (1999) it can be assumed that this is also a good reference spectral class for our sample. Late type giants also show a strong $\mathrm{SiO}$ band around $8 \mu \mathrm{m}$. The Engelke function does not contain this molecular absorption band, so it was modified by SP to correct for its presence. They used a median $\mathrm{SiO}$ profile taken from LRS spectra of naked giants. We also modify the Engelke function, by taking the absorption profile from MARCS theoretical modelling for a $3500 \mathrm{~K} \mathrm{M}$ giant. The feature was scaled to get the same absorption maximum $(15 \%)$ as was used in SP. The modified Engelke function was then used to subtract the stellar continuum from all our individual spectra.

To quantify the shape of the dust emission features, SP examined the flux densities at 10, 11 and $12 \mu \mathrm{m}$. A "silicate" dust sequence can be seen when plotting $F_{10} / F_{12}$ as a function of $F_{10} / F_{11}$. Figure 4 shows these flux ratios for our sample. Our sources follow the same empirical power law that SP fitted to their data: $F_{10} / F_{12}=1.32\left(F_{10} / F_{11}\right)^{1.77}$. Thus, our Bulge 

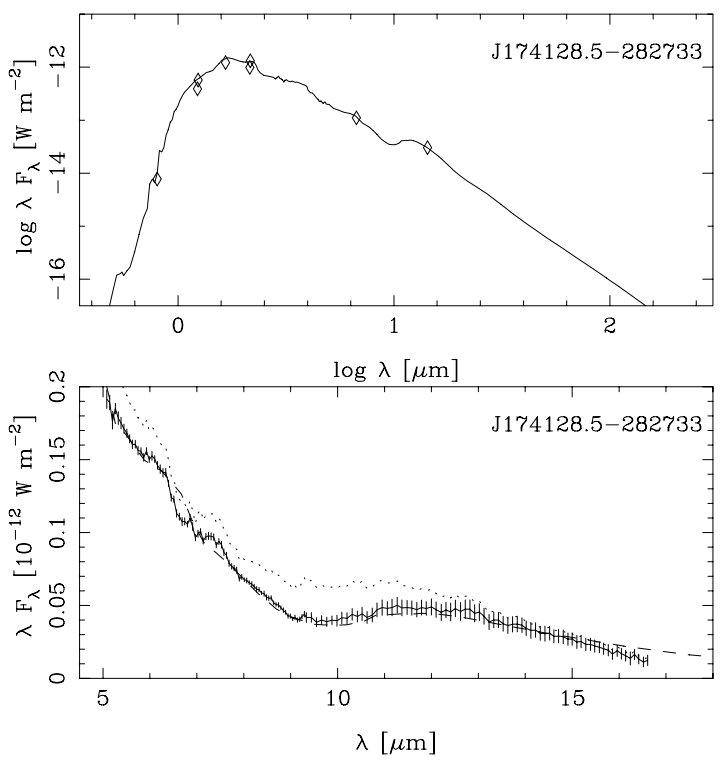

Fig. 2. Model fit to the DENIS, 2MASS and ISOGAL photometry and spectra of source J174128.5-282733. The upper plot gives the unreddened photometry (diamonds) and the reddened model fit (full line). The lower plot shows the observed spectrum (full line) with error bars at each wavelength point of the CAM CVF. The dotted line is the spectrum after correction for the interstellar extinction. The dashed line gives the best fit to observed spectrum. The above figure is given as an example. The same plot is available for all sources in the electronic form.

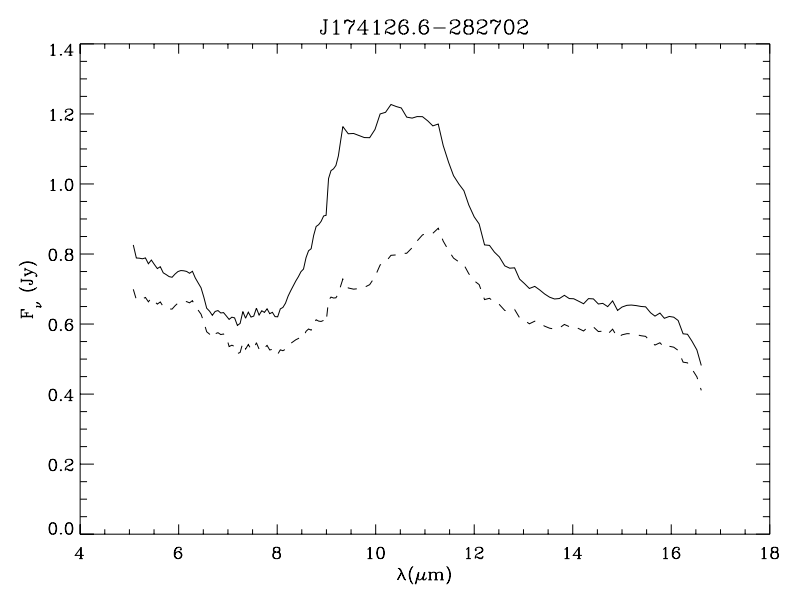

Fig. 3. An example of the effect of the extinction correction applied to the spectrum of a source in the C32 field $\left(A_{\mathrm{V}}=6.3\right)$. The dashed line shows the observed spectrum, the solid one that after correction for extinction (see text).

sample of AGB stars follows the same "silicate" dust sequence as was found for a solar neighbourhood sample of Long Period Variables.

SP use the flux ratios to classify the dust spectra. They take the position on the power law that is closest to the actual data point (along a line orthogonal to the power-law curve) and from this determine a flux ratio $F_{11} / F_{12}$. A "silicate" emission index $\left.n=10\left(F_{11} / F_{12}\right)-7.5\right)$ is then defined to classify the dust spectrum. SP's Fig. 3 shows examples for each silicate emission index, ranging from 1 to 8 . Index 1 corresponds to the broad emission feature, which peaks beyond $11 \mu \mathrm{m}$. The largest index 8 represents the narrower silicate emission feature with a peak at approximately $10 \mu \mathrm{m}$. Our sources have indices ranging from 1 to 5 (see Table 3 ). This indicates that our stars are dominated by

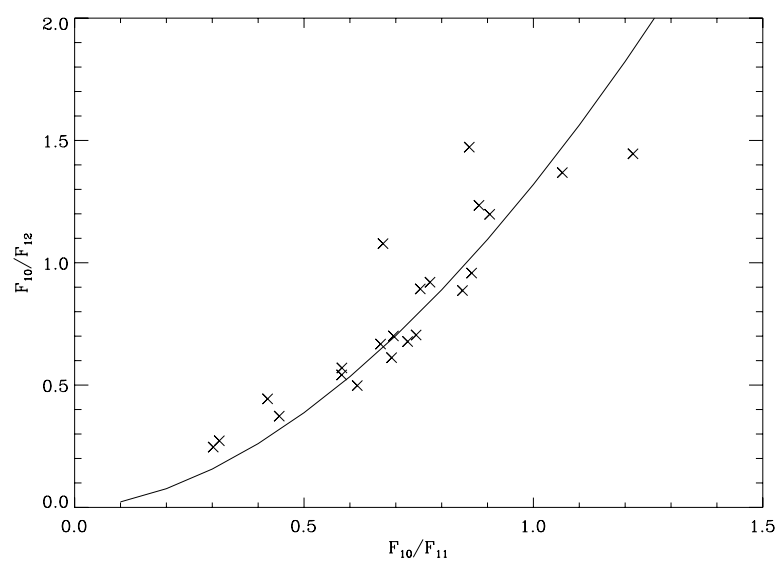

Fig. 4. The silicate dust sequence, as defined in Sloan \& Price (1995). The power law was a fit to their sample and is also a good representation for the dust features seen in our sample.

the "broader" type of dust feature and do not contain the narrow features connected to the classical silicate dust. We discuss this further in the modelling of the spectra in the next section.

\section{Modelling}

The radiative transfer model of Groenewegen (1993; also see Groenewegen 1995) is used. This model was developed to handle non- $r^{-2}$ density distributions in spherically symmetric dust shells. It simultaneously solves the radiative transfer equation and the thermal balance equation for the dust.

When the input spectrum of the central source is fixed, the shape of the SED is exclusively determined by the dust optical depth, defined by:

$$
\begin{aligned}
\tau_{\lambda} & =\int_{r_{\text {inner }}}^{r_{\text {outer }}} \pi a^{2} Q_{\lambda} n_{\mathrm{d}}(r) \mathrm{d} r \\
& =5.405 \times 10^{8} \frac{\dot{M} \Psi Q_{\lambda} / a}{R_{\star} v_{\infty} \rho_{\mathrm{d}} r_{\mathrm{c}}} \int_{1}^{x_{\max }} \frac{R(x)}{x^{2} w(x)} \mathrm{d} x
\end{aligned}
$$

where $x=r / r_{\mathrm{c}}, \dot{M}(\mathrm{r})=\dot{M} R(x)$ and $v(r)=v_{\infty} w(x)$. The normalized mass-loss rate profile $R(x)$ and the normalized velocity law $w(x)$ should obey $R(1)=1$ and $w(\infty)=1$, respectively. In the case of a constant mass-loss rate and a constant velocity, the integral in Eq. (1) is essentially unity since $x_{\max }$ is typically much larger than 1 . The symbols and units in Eq. (1) are: the (presentday) mass-loss rate $\dot{M}$ in $M_{\odot} / \mathrm{yr}, \Psi$ the dust-to-gas mass ratio (assumed constant with radius), $Q_{\lambda}$ the extinction efficiency, $a$ the grain size in cm (the model assumes a single grain size), $R_{\star}$ the stellar radius in $R_{\odot}, v_{\infty}$ the terminal velocity of the dust in $\mathrm{km} \mathrm{s}^{-1}$, $\rho_{\mathrm{d}}$ the dust grain specific density in $\mathrm{g} \mathrm{cm}^{-3}, r_{\mathrm{c}}$ the inner dust radius in units of stellar radii and $x_{\max }$ the outer radius in units of $r_{\mathrm{c}}$.

In the present model calculations the outer radius is set at the distance where the dust temperature equals $20 \mathrm{~K}$, corresponding to a few thousand inner radii. We assume a dust-to-gas ratio of $\Psi=0.005$ (see e.g. Heras \& Hony 2005, who also showed that there is significant scatter around this mean value), a grain specific density of $\rho_{\mathrm{d}}=3.0 \mathrm{~g} \mathrm{~cm}^{-3}$ (a compromise between a typical density for amorphous silicates of $3.3 \mathrm{~g} \mathrm{~cm}^{-3}$, e.g. Draine \& Lee 1984; and a density of about $2.5 \mathrm{~g} \mathrm{~cm}^{-3}$ for amorphous alumina, e.g. Begemann et al. 1997), a constant mass-loss rate and a constant outflow velocity of $10 \mathrm{~km} \mathrm{~s}^{-1}$. The sources have been put at a distance of $8.5 \mathrm{kpc}$. 


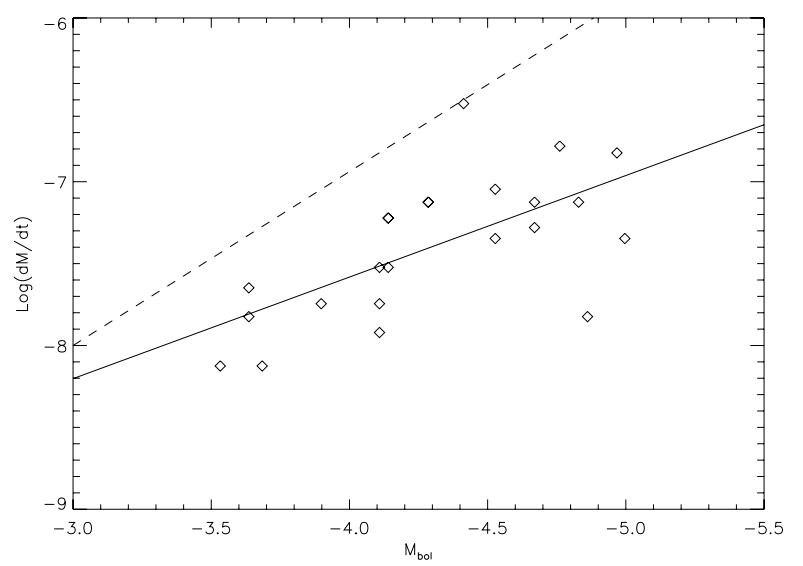

Fig. 5. Mass-loss rates in units of $M_{\odot} / \mathrm{yr}$ plotted against $M_{\text {bol }}$. The solid line is a linear fit for our stars and modelling. The dashed line is the linear fit by $\mathrm{ABC}$ to SRVs in the Bulge and extreme mass-losing stars in the LMC.

The remaining free parameters of the model are the massloss rate, the luminosity and the dust condensation temperature, which was adopted to be $1500 \mathrm{~K}$, as most stars are dominated by aluminium oxide dust which is believed to condense first in an oxygen-rich environment (e.g. Salpeter 1977; Tielens 1990).

For the central stars the photospheric spectra of O-rich stars from Fluks et al. (1994) have been adopted. They have temperatures from $T_{\text {eff }}=3850 \mathrm{~K}$ (for spectral type M0), $3297 \mathrm{~K}$ (M6), to $2500 \mathrm{~K}$ (M10). It is important to remark that with the present data and the relatively high extinction we cannot well constrain the $T_{\text {eff }}$ so that the temperature values given in Table 3 are uncertain. The photospheric models are for solar metallicity only and therefore may not be entirely appropriate for the sample under consideration. The photospheric spectra of O-rich stars show strong molecular absorption features. The Fluks spectra contain absorption features around $5 \mu \mathrm{m}$ due to $\mathrm{CO}$ and $\mathrm{SiO}$ and also some absorption around $8 \mu \mathrm{m}$ (caused by the $\mathrm{SiO}$ fundamental band). The Fluks models do not include $\mathrm{H}_{2} \mathrm{O}$ absorption around $6 \mu \mathrm{m}$. The depth of the molecular absorption features is dependent on different parameters such as temperature, metallicity, gravity and mass of the star. The situation is even more complicated in the case of the unstable atmospheres of pulsating stars, such as our sample of AGB stars, which demand dynamical modelling. When choosing the Fluks photospheric model to use we have selected the $T_{\text {eff }}$ that gives the best overall fit to the spectrum between 5 and $8 \mu \mathrm{m}$, allowing for discrepancies where the molecular absorption bands are found.

The types of dust used are combinations of silicate (from David \& Pégourié 1995) and amorphous aluminium oxide (porous $\mathrm{Al}_{2} \mathrm{O}_{3}$ from Begemann et al. 1997, as this seems to provide a better fit than compact amorphous aluminium oxide; see e.g. Lorenz-Martins \& Pompeia 2000). From the optical constants provided in these papers ${ }^{1}$ the absorption coefficients are calculated in the Rayleigh limit of small particles. Initially, pure silicate and pure $\mathrm{Al}_{2} \mathrm{O}_{3}$ dust were used in the modelling but this resulted in unsatisfactory fits. Subsequently, linear combinations of the absorption coefficients were used. This assumes that the two species coexist with the same temperature structure. More complicated situations, such as core-mantle grains or different spatial density distributions have not been considered.

${ }^{1}$ For aluminium oxide, constant values of $n=1.5$ and $k=0.01$ have been adopted for wavelengths below 5.4 micron while between 5.4 micron and the first available datapoint at 7.8 micron the optical constants have been linearly interpolated.
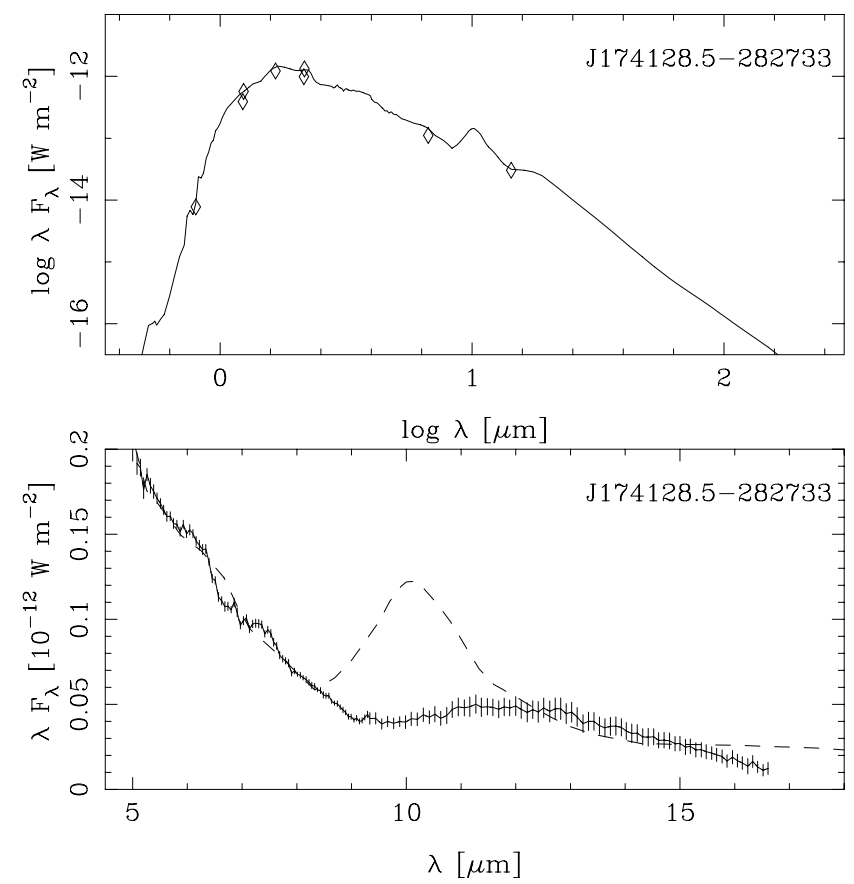

Fig. 6. An example of the spurious result found by fitting a model with pure silicate dust to a source taken from our sample. The mass-loss rate needs to be twice as high as when fitting with pure alumina dust. The strong silicate emission feature seen in the model is not, of course, present in the observed spectrum. Photometry alone does not reveal this. The fit to the same star of the photometry and the favoured pure alumina dust spectrum can be seen in Fig. 2.

The dielectric constants for silicate have been derived by David \& Pégourié (1995) from analysis of IRAS LRS spectra; in that sense it is an "astronomical silicate". An alternative approach is to use the dielectric constants measured in the laboratory for several species (spinel, melilite, olivines, pyroxenes) and fit all of these to the mid-IR spectra (e.g. Heras \& Hony 2005). In view of the uncertainties regarding the spatial location and temperature distribution of these various species and the possibility that they form complicated core-mantle grains we opted here to use a general type of silicate.

Thus, in our modelling we only make use of amorphous alumina or silicate dust and avoid other components such as melilite $\left(\mathrm{CaAl}_{2} \mathrm{SiO}_{7}\right)$. Considering the limited spectral resolution, sensitivity and wavelength range available of the CVF, it would be very difficult to make a distinction between silicate dust and melilite as both peak around $9.5 \mu \mathrm{m}$. The difference between the two becomes more detectable when observing in the 17-20 $\mu \mathrm{m}$ range (longward of the ISOCAM CVF coverage), where they show a distinct behaviour. We also looked at the effect of using another type of silicate than the astronomical silicate in our modelling. For this we used the extinction efficiencies of olivine $\left(\mathrm{Mg}_{0.8} \mathrm{Fe}_{1.2} \mathrm{SiO}_{4}\right)$, taken from de Database of Optical Constants of the Laboratory Astrophysics Group of the AIU Jena. Modelling of a source with this alternative silicate did not show an important impact on our results.

In order to compare the models directly to the observations they have been reddened using the $A_{\mathrm{V}}$ 's listed in Table 2 . In Table 3, the resulting luminosities, mass-loss rates and the fraction of alumina dust are given. A number of sources have relatively high luminosities $\left(>5000 L_{\odot}\right)$ but no detectable mass loss. These are likely to be sources in the foreground of the Bulge. Figure 2 shows the fits to the photometry data and the spectra for all sources. 
Table 3. Results from the modelling. Mass-loss rates of $10^{-9} M_{\odot} / \mathrm{yr}$ or below are not detectable in our spectra. Sources without mass loss and $L_{*}>5000 L_{\odot}$ are likely to be foreground sources. Also indicated is the SE class according to the SP-definition. For each source we give the $T_{\text {eff }}$ used in the modelling but this temperature is not well constrained with the available data and is uncertain.

\begin{tabular}{|c|c|c|c|c|c|}
\hline ISOGAL name & $\begin{array}{c}\text { luminosity } \\
L_{\odot} \\
\end{array}$ & $\begin{array}{c}T_{\text {eff }} \\
\mathrm{K} \\
\end{array}$ & $\begin{array}{c}\text { mass-loss rate } \\
M_{\odot} / \mathrm{yr}\end{array}$ & $\begin{array}{c}\text { alumina fraction } \\
\%\end{array}$ & $\begin{array}{c}\text { remark } \\
\text { /SE-class }\end{array}$ \\
\hline \multicolumn{6}{|l|}{$C 32$} \\
\hline $\mathrm{J} 174121.4-282810$ & 6300 & 3750 & $\leq 1.0 E-9$ & - & possible foreground \\
\hline $\mathrm{J} 174123.6-282723$ & 2800 & 3129 & $1.8 \mathrm{E}-8$ & 40 & 5 \\
\hline $\mathrm{J} 174124.7-282801$ & 3400 & 3550 & $1.8 \mathrm{E}-8$ & 60 & 6 \\
\hline $\mathrm{J} 174125.7-282807$ & 4000 & 3129 & $7.5 \mathrm{E}-8$ & 60 & 4 \\
\hline $\mathrm{J} 174126.6-282702$ & 4500 & 2500 & $3.0 \mathrm{E}-7$ & 40 & 5 \\
\hline $\mathrm{J} 174127.3-282851$ & 6200 & 2500 & $1.7 \mathrm{E}-7$ & 90 & 1 \\
\hline $\mathrm{J} 174128.5-282733$ & 7700 & 3129 & $4.5 \mathrm{E}-8$ & 100 & 0 \\
\hline $\mathrm{J} 174130.2-282801$ & 1700 & 2890 & $\leq 1.0 E-9$ & - & - \\
\hline $\mathrm{J} 174131.2-282815$ & 6800 & 3129 & $1.5 \mathrm{E}-8$ & 60 & 3 \\
\hline $\mathrm{J} 174133.7-282723$ & 3400 & 3490 & $1.2 \mathrm{E}-8$ & 80 & 4 \\
\hline $\mathrm{J} 174134.1-282653$ & 5500 & 3550 & $\leq 1.0 E-9$ & - & possible foreground \\
\hline \multicolumn{6}{|l|}{ C35 } \\
\hline 174941.1-291921 & 2000 & 3129 & $7.5 \mathrm{E}-9$ & 100 & - \\
\hline J174942.3-292043 & 3500 & 3129 & $6.0 \mathrm{E}-8$ & 90 & 2 \\
\hline J174943.3-291947 & 7500 & 3129 & $1.5 \mathrm{E}-7$ & 40 & 5 \\
\hline J174943.7-292154 & 5000 & 3129 & $4.5 \mathrm{E}-8$ & 100 & 2 \\
\hline J174944.5-292009 & 3500 & 3129 & $6.0 \mathrm{E}-8$ & 100 & 0 \\
\hline 174946.1-291944 & 3400 & 3129 & $3.0 \mathrm{E}-8$ & 100 & 1 \\
\hline J174946.4-292005 & 4000 & 3129 & $7.5 \mathrm{E}-8$ & 80 & 2 \\
\hline J174946.5-291917 & 2200 & 3129 & $2.3 \mathrm{E}-8$ & 100 & 6 \\
\hline J174946.5-291933 & 6600 & 3129 & $7.5 \mathrm{E}-8$ & 100 & 2 \\
\hline J174948.1-292104 & 2200 & 3129 & $1.5 \mathrm{E}-8$ & 100 & 0 \\
\hline J174949.2-291932 & 5000 & 3129 & 9.0 E-8 & 80 & 2 \\
\hline J174951.7-292108 & 3500 & 3129 & $3.0 \mathrm{E}-8$ & 80 & 3 \\
\hline \multicolumn{6}{|l|}{$O G L E$} \\
\hline J175511.9-294027 & 1900 & 3397 & $\leq 1.0 E-9$ & - & - \\
\hline J175514.1-293928 & 2300 & 3129 & 7.5 E-9 & 100 & 0 \\
\hline J175514.9-293918 & 6200 & 3397 & $\leq 1.0 E-9$ & - & possible foreground \\
\hline J175515.3-294016 & 5700 & 2500 & $7.5 \mathrm{E}-8$ & 100 & 1 \\
\hline J175518.9-294142 & 5700 & 2890 & $5.3 \mathrm{E}-8$ & 70 & 3 \\
\hline $\mathrm{J} 175521.0-294055$ & 2400 & 3490 & $\leq 1.0 E-9$ & - & - \\
\hline
\end{tabular}

\section{Discussion}

The luminosities derived for our sample range from 1700 to $7700 L_{\odot}$ with a mean of $4100 L_{\odot}$, for the assumed distance of $8.5 \mathrm{kpc}$. By comparing the period distribution of Bulge Miras with predictions from synthetic AGB evolutionary models, Groenewegen \& Blommaert (2005) found that the initial masses of the Miras fall in a small range of 1.5 to $2 M_{\odot}$. The AGB models by Vassiliadis \& Wood (1993) give a minimum luminosity at the Thermally Pulsing AGB of $\approx 1600 L_{\odot}$ for both a 1.5 and a $2 M_{\odot}$ initial mass star and $7000 L_{\odot}$ as the maximum luminosity. The luminosities of our sample are in agreement with such a range, certainly when taking into account the effect of variability on the AGB. One cannot exclude that our sample also contains smaller mass objects. A $1 M_{\odot}$ AGB star has, according to the Vassiliadis \& Wood (1993) models, luminosities in the range of 1370 to $3100 L_{\odot}$ and so covers the lower range but would not be able to explain the higher luminosity part in our sample.

ABC studied the ISOGAL sources in the Baade's window. They modelled the spectral energy distributions obtained by combining optical, near-infrared and ISOGAL magnitudes. The modelling was done using the radiative transfer code DUSTY (Ivezić et al. 1999). Figure 5 shows the mass-loss rates obtained with our modelling as function of $M_{\mathrm{bol}}$. We see a trend of increasing mass-loss rates for higher luminosities. When comparing our mass-loss rates with those obtained in $\mathrm{ABC}$ we find that in the same luminosty range our mass-loss rates are on average about a factor of five lower. Also the relation between the mass-loss rate and luminosity has a different slope. $\mathrm{ABC}$ included the mass-loss rates and luminosities of extreme mass-losing AGB stars in the LMC from van Loon et al. 1999. The latter group contains both oxygen and carbon-rich stars. The LMC stars lie on the extension of the sequence formed by the Bulge stars. They derived a linear fit to the mass-loss rates and the absolute bolometric magnitudes and found $\mathrm{d} M / \mathrm{d} t \propto L^{2.7}$ whereas we find $\mathrm{d} M / \mathrm{d} t \propto L^{1.55}$ for our sample.

There are a some differences between the modelling used in $\mathrm{ABC}$ and that presented in this paper. We use the same gas-todust ratio (200) and dust particle specific density $\left(3 \mathrm{~g} \mathrm{~cm}^{-3}\right)$ but $\mathrm{ABC}$ uses pure silicate dust, a lower dust condensation temperature (1000 K versus $1500 \mathrm{~K}$ in our modelling) and also a higher expansion velocity. As no measurements of the expansion velocities for these Bulge stars exist we assumed the same velocity for all objects: $10 \mathrm{~km} \mathrm{~s}^{-1}$ (see Sect. 6). In ABC the expansion velocity is an outcome of the modelling in DUSTY. The velocities are in general higher, in the order of $15-16 \mathrm{~km} \mathrm{~s}^{-1}$. These values are rather high for low mass-loss rate sources, so that we decided to continue using the lower value. Increasing the expansion velocities in our models by 1.5 would increase the mass-loss rates by the same factor (see Eq. (1)). Lowering the condensation temperature from 1500 to $1000 \mathrm{~K}$ changes the inner dust radius and would increase the mass-loss rates by about a factor 2.5. The remaining discrepancy comes from the different 
dust species used. The dust opacities are lower for the pure silicate dust used in $\mathrm{ABC}$ than for that containing different fractions of alumina as in our analysis (ABC: $\chi_{60}=70 \mathrm{~cm}^{2} \mathrm{~g}^{-1}$ versus our $\chi_{60}=137 \mathrm{~cm}^{2} \mathrm{~g}^{-1}$ for pure alumina dust and $\chi_{60}=90 \mathrm{~cm}^{2} \mathrm{~g}^{-1}$ for a mixture of $40 \%$ alumina and $60 \%$ silicate), leading to an average discrepancy of about 1.5. However, the difference in mass loss is not only due to this scaling of the absolute opacity as this does not take into account the difference in spectral features that also influence how a fit is performed in practice.

To investigate this further we also modelled two of the ABC SEDs and found the same discrepancy. We only reach the same higher mass-loss rates as $\mathrm{ABC}$ when using the same expansion velocity and dust condensation temperature but more importantly also the same type of dust: pure silicates. This can be explained by comparing the wavelength dependence of the opacities of the amorphous alumina and silicate dust. In the latter there are strong emission features peaking at 9.7 and $18 \mu \mathrm{m}$. Both these features fall only marginally within the $15 \mu \mathrm{m}$ ISOCAM band (12-18 $\mu \mathrm{m})$, contrary to the alumina dust emission feature which peaks around $12 \mu \mathrm{m}$ and is very broad, covering the range $10-20 \mu \mathrm{m}$, which goes well beyond the limits of the ISOCAM $15 \mu \mathrm{m}$ filter. To explain the excess observed in the $15 \mu \mathrm{m}$ band one thus needs considerably higher mass-loss rates in the case of silicate dust than for alumina, as is shown in Fig. 6, amounting to about a factor of two for the case shown. This comparison demonstrates the importance of including spectroscopic data when modelling the SEDs of the mass-losing AGB stars.

We do not find that the LMC AGB stars are simply on the extension of the sequence of the mass-losing SRVs in the Bulge as ABC did (cf. their Fig. 9). The extrapolation of our sequence rather leads to a relationship of mass-loss rates versus luminosities as observed for the supergiants.

\subsection{Mass-loss rates versus colour}

OOS discuss the usage of ISOGAL colours to investigate the mass-loss rates of the AGB stars detected in different Bulge fields. In Fig. 7 we plot the mass-loss rates, resulting from our modelling, with the observed ISOGAL colours: $K_{\mathrm{s}, \mathrm{o}}-[7]$, $K_{\mathrm{s}, \mathrm{o}}-$ [15] and [7]-[15]. It is clear that for the low mass-loss rates in our sample, $K_{\mathrm{s}, \mathrm{o}}-[7]$ is not such a good discriminator since sources with mass-loss rates below $10^{-7} M_{\odot} /$ yr cluster around $K_{\mathrm{s}, \mathrm{o}}-[7] \approx 0.5$. This wavelength range is dominated by the photospheric colours, which are hardly influenced by the relatively low dust content. Clearly one needs the [15] band where the excess emission is much more apparent.

In their analysis of the total mass-loss rate in the bulge, OOS use a theoretical relation for mass-loss rates versus the $K_{\text {s,o }}-$ [15] colour derived by Jeong et al. (2003). The model concerned is based on a consistent time-dependent treatment of hydrodynamics, thermodynamics, equilibrium chemistry and dust formation. However, no details are given about the effect of dust type on mass-loss rates. Our derived mass-loss rates clearly do not follow this law which has a much steeper change in massloss rate as a function of $K_{\mathrm{s}, \mathrm{o}}-$ [15] colour.

\subsection{The dust content}

As discussed in the introduction, different dust species are seen in the outflows of AGB stars. It is generally believed that aluminium oxide dust would be first to form at a temperature of about $1500 \mathrm{~K}$. The presence of amorphous alumina dust can be seen through a broad feature that peaks around
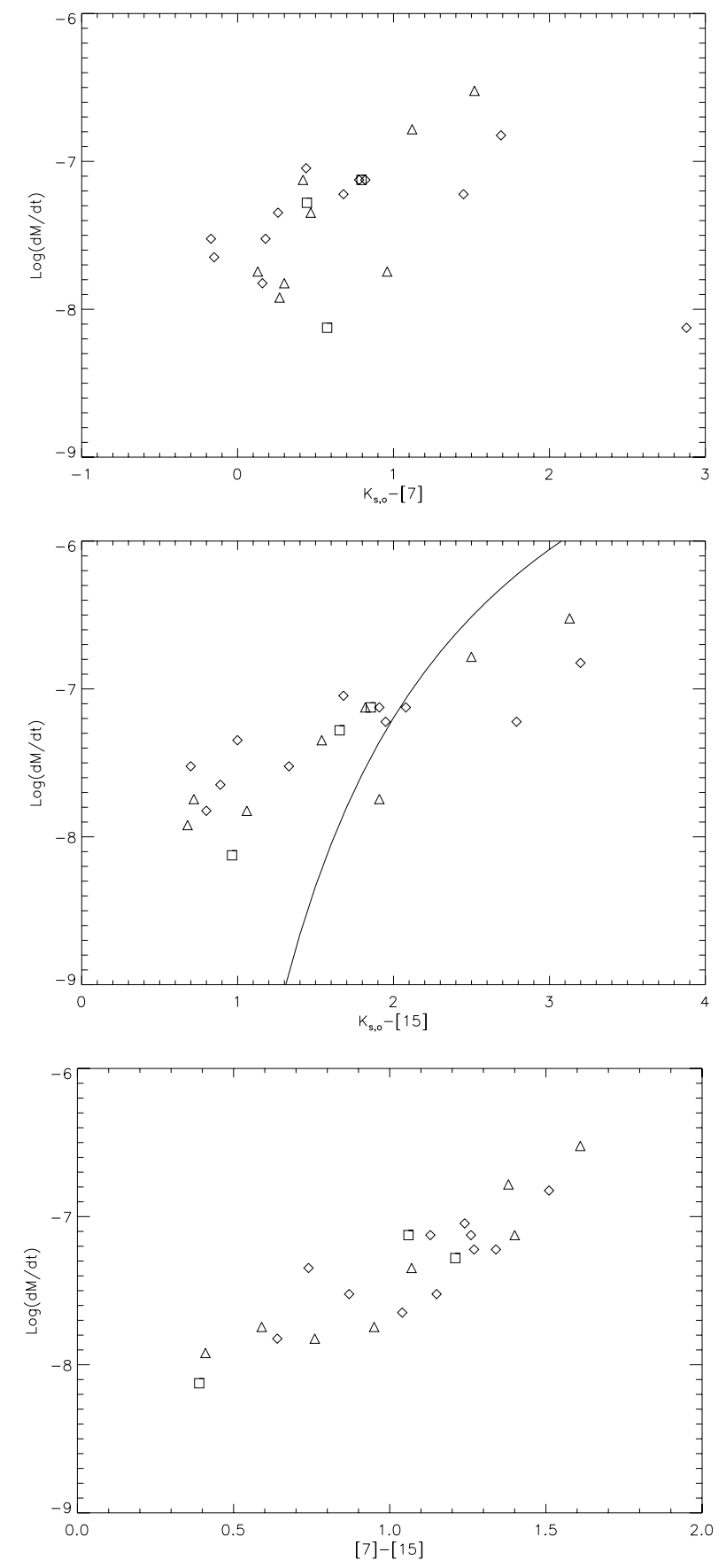

Fig. 7. Mass-loss rates in units of $M_{\odot} / \mathrm{yr}$ plotted against ISOGAL colours. Diamonds represent sources from the C35 field, triangles: C32 and squares the OGLE field. The solid line is the relation between $K_{\mathrm{s}, \mathrm{o}}-$ [15] and mass-loss rate according to Jeong et al. (2003).

11-12 $\mu \mathrm{m}$. Amorphous silicates form at lower temperatures and show a spectral feature peaking around $9.7 \mu \mathrm{m}$. One would expect that in a dust-forming region of low density in the outer regions of a stellar atmosphere, associated with a low massloss rate, the dominant dust species would be alumina and only in regions of higher density would further dust types such as the amorphous silicates be formed, as found for a sample of LMC AGB stars by Dijkstra et al. (2005). In comparison with most studies so far, our sample is dominated by relatively low mass-loss rate $\left(<10^{-7} M_{\odot} / y r\right)$ stars and so we expect to find a large fraction where the dust content is dominated by alumina dust. Indeed we find that $70 \%$ of our sample has a low silicate 
content ( $\leq 20 \%$, see Table 3$)$. Contrary to Heras \& Hony (2005) and Speck et al. (2000) we find a large fraction of sources where we can fit the SED with pure amorphous alumina, whereas they do not find evidence for any such cases. The mass-loss rates for our sample are lower than those in Heras \& Hony (2005) which could be the reason for this different result. No massloss rates are given in Speck et al. (2000). The difference may also be caused by the uncertainty in estimating the $\mathrm{SiO}$ absorption feature $(7.5-11 \mu \mathrm{m})$ which overlaps in wavelength with the silicate emission feature. The strength of the $\mathrm{SiO}$ band is difficult to estimate and is rather weak in the Fluks models that we used to model the photospheric contribution. Underestimating the $\mathrm{SiO}$ band may lead to an underestimate of features peaking at $10 \mu \mathrm{m}$ such as melilite or silicate. Mid-infrared spectra with higher sensitivity and resolution and a broader wavelength coverage would be needed to investigate the presence of other dust features than the alumina species, but even so, their contribution can only be small.

\section{Conclusions}

We have presented the ISOCAM CVF spectra of a sample of AGB stars previously detected in the ISOGAL survey. These are the first mid-IR spectra of Bulge AGB stars with mass-loss rates down to $10^{-8} M_{\odot} / \mathrm{yr}$. Modelling of the ISOGAL photometry and the CVF spectra show that our sample consists of AGB stars with luminosities between 1700 to $7700 L_{\odot}$, in agreement with the range of luminosities of Thermally Pulsing AGB stars with initial masses of 1.5 to $2 M_{\odot}$. The mass-loss rates of our sample are in the range of $10^{-8}-5 \times 10^{-7} M_{\odot} / \mathrm{yr}$. We have demonstrated that the availability of the mid-IR spectra, which allow the identification of the circumstellar dust type, plays an important role in determining accurate dust mass-loss rates. Our sample, that mainly contains low mass-loss rate stars, has a dust content that is dominated by amorphous aluminium oxide dust.

Acknowledgements. The ISOCAM data presented in this paper were analysed using "CIA", a joint development by the ESA Astrophysics Division and the ISOCAM Consortium. The ISOCAM Consortium is led by the ISOCAM PI, C. Cesarsky. This research made use of the SIMBAD database, operated at CDS, Strasbourg, France. We thank Leen Decin for providing us a MARCS model. J.B. thanks Sacha Hony for useful discussions on the dust formation around AGB stars. I.S.G. acknowledges the receipt of a travel grant from the CNRS-NRF scheme. We thank the referee, Dr. A. Speck, for her constructive comments.

\section{References}

Alard, C., Blommaert, J. A. D. L., Cesarsky, C., et al. 2001, ApJ, 552, 289 (ABC) Begemann, B., Dorschner, J., Henning, Th., et al. 1997, ApJ, 476, 199 Blommaert, J. A. D. L., Okumura, K., \& Boulanger, F. 2001, ISOCAM Photometry, in The Calibration Legacy of the ISO Mission, ed. L. Metcalfe, A. Salama, S. Peschke, \& M. Kessler, ESA SP-481, 77
Blommaert, J. A. D. L., Siebenmorgen, R., Coulais, A., et al. 2003, The ISO Handbook, Volume II - CAM - The ISO Camera Version 2.0 (June, 2003) Series, ed. T. G. Mueller, J. A. D. L. Blommaert, \& P. Garcia-Lario, ESA SP-1262

Cardelli, J. A., Clayton, G. C. \& Mathis, J. S. 1989, ApJ, 345, 245

Cesarsky, C. J., Abergel, A., Agnese, P., et al. 1996, A\&A, 315, L32

Coulais, A., \& Abergel, A. 2000, A\&AS, 141, 533

Cutri, R. M., Skrutskie, M. F., Van Dyk, S., et al. 2003, Explanatory Supplement to the 2MASS All-Sky Data Release

David, P., \& Pégourié, B. 1995, A\&A, 293, 833

Dijkstra, C., Speck, A. K., Reid, R. B., \& Abraham, P. 2005, ApJ, 633, L133

Draine, B. T. 1985, ApJS, 57, 587

Draine, B. T., \& Lee, H. M. 1984, ApJS, 285, 89

Engelke, C. W. 1992, AJ, 104, 1248

Epchtein, N., Deul, E., Derriere, S., et al. 1999, A\&A, 349, 236

Fluks, M. A., Plez, B., Thé, P. S., et al. 1994, A\&AS, 105, 311

Glass, I. S., Ganesh, S., Alard, C., et al. 1999, MNRAS, 308, 127

Groenewegen, M. A. T. 1993, Ph.D. Thesis, Chap. 5, University of Amsterdam Groenewegen, M. A. T. 1995, A\&A, 293, 463

Groenewegen, M. A. T., \& Blommaert, J. A. D. L. 2005, A\&A, 443, 143

Habing, H. J. 1996, A\&ARv, 7, 97

Heras, A. M., \& Hony, S. 2005, A\&A, 439, 171

Hron, J., Aringer, B., \& Kerschbaum, F. 1997, A\&A, 322, 280

Ivezić, Z., Nenkova, M., \& Elitzur, M. 1999, User's Manual for DUSTY, [arXiv:astro-ph/9910475]

Jeong, K. S., Winters, J. M., Le Bertre, T., \& Sedlmayr, E. 2003, Theoretical color mass-loss relations for O-rich Mira stars, in Mass-Losing Pulsating Stars and their Circumstellar Matter, ed. Y. Nakada, M. Honma, \& M. Seki, ASSL, 283, 139

Little-Marenin, I. R., Little, S. J., Stencel, R. E., \& Nuth, III, J. A. 1989, BAAS, 21,1118

Little-Marenin, I. R., \& Little, S. J. 1990, AJ, 99, 1173

Lloyd-Evans, T. 1976, MNRAS, 174, 169

Lorenz-Martins, S., \& Pompeia, L. 2000, MNRAS, 315, 856

Ojha, D. K., Omont, A., Schuller, F., et al. 2003, A\&A, 403, 141 (OOS)

Okumura, K., Pérault, M., \& Longval, Y. 1998, Ghosts in ISOCAM images, ISO technical report, http://www.iso.vilspa.esa.es/

Omont, A., Ganesh, S., Alard, C., et al. 1999, A\&A, 348, 755

Omont, A., Gilmore, G. F., Alard, C., et al. 2003, A\&A, 403, 975

Onaka, T., de Jong, T., \& Willems, F. J. 1989, A\&A, 218, 169

Ott, S., et al. 1997, in Astronomical Data Analysis Software and Systems (ADASS) VI, ed. G. Hunt, \& H. E. Payne (San Francisco: ASP), ASP Conf. Ser., 125,34

Rich, R. M., \& Origlia, L. 2005, ApJ, 634, 1293

Salpeter, E. E. 1977, ARA\&A, 15, 267

Schuller, F., Ganesh, S., Messineo, M., et al. 2003, A\&A, 403, 955

Schultheis, M., Ganesh, S., Simon, G., et al. 1999, A\&A, 349, L69

Sloan, G. C., \& Price, S. D. 1995, ApJ, 451, 758 (SP)

Sloan, G. C., \& Price, S. D. 1998, ApJS, 119, 141

Speck, A. K., Barlow, M. J., Sylvester, R. J., \& Hofmeister, A. M. 2000, A\&AS, 146,437

Stencel, R. E., Nuth, III, J. A., Little-Marenin, I. R., \& Little, S. J. 1990, ApJ, 350, L45

Tielens, A. G. G. M. 1990, in From Miras to planetary nebulae: Which path for stellar evolution? (Gif-sur-Yvette, France: Éditions Frontières), 186

Udalski, A., Szymański, M., Kubiak, M., et al. 2002, AcA, 52, 217

van Loon, J. Th., Groenwegen, M. A. T., de Koter, A., et al. 1999, A\&A, 351, 559

Vassiliadis, E., \& Wood, P. R. 1993, ApJ, 413, 641

Zoccali, M., Renzini, A., Ortolani, S., et al. 2003, A\&A, 399, 931 
J. A. D. L. Blommaert et al.: ISO Mid-IR spectroscopy of Galactic Bulge AGB stars, Online Material p 1

\section{Online Material}


J. A. D. L. Blommaert et al.: ISO Mid-IR spectroscopy of Galactic Bulge AGB stars, Online Material p 2
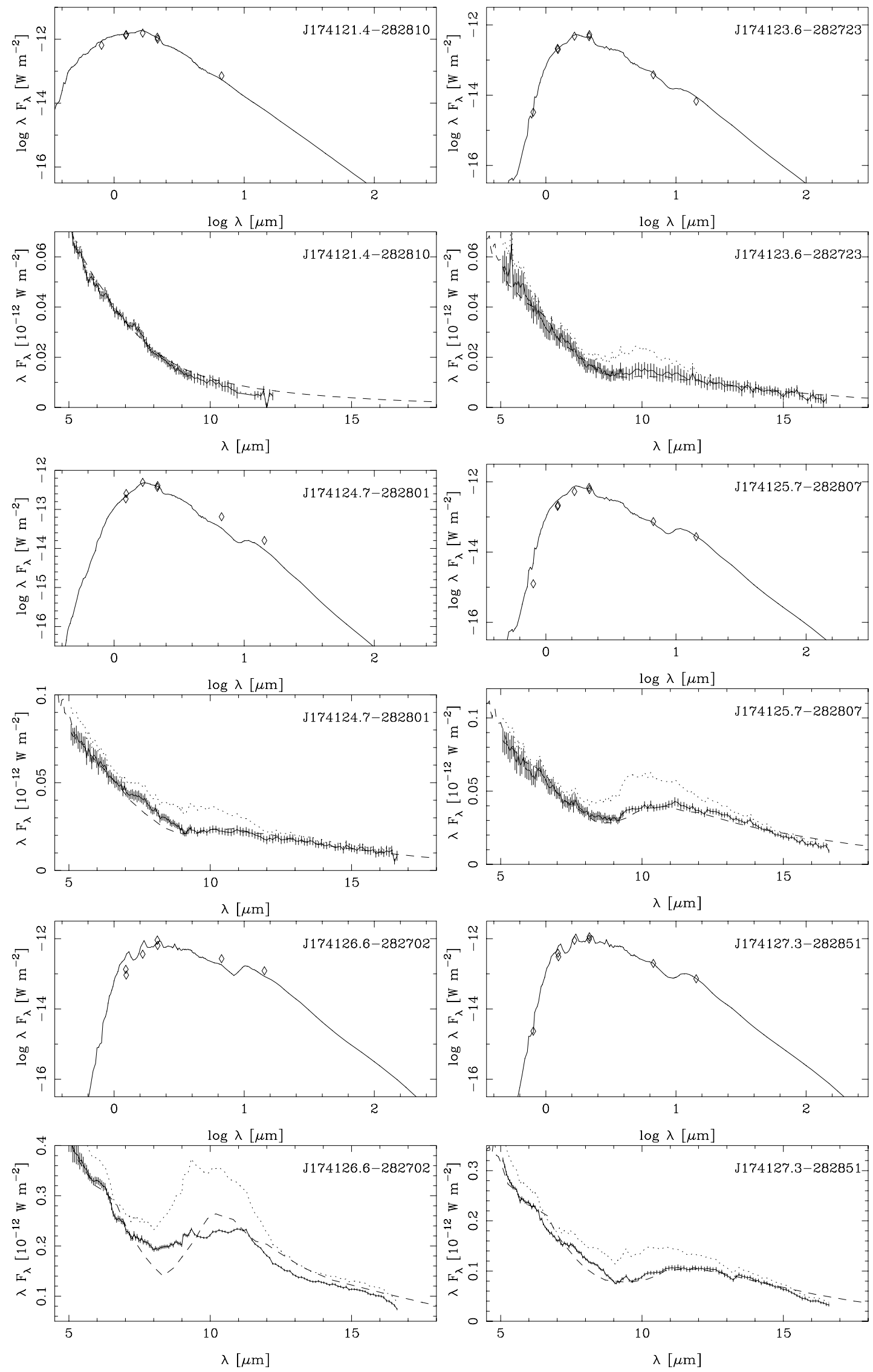

Fig. 2. Model fits to the DENIS, 2 MASS and ISOGAL photometry and spectra. For each source there are two plots. The upper one gives the unreddened photometry (diamonds) and the reddened model fit (full line). The lower plot shows the observed spectrum (full line) with error bars at each wavelength point of the CAM CVF. The dotted line is the spectrum after correction for the interstellar extinction. The dashed line gives the best fit to observed spectrum. 
J. A. D. L. Blommaert et al.: ISO Mid-IR spectroscopy of Galactic Bulge AGB stars, Online Material $p 3$
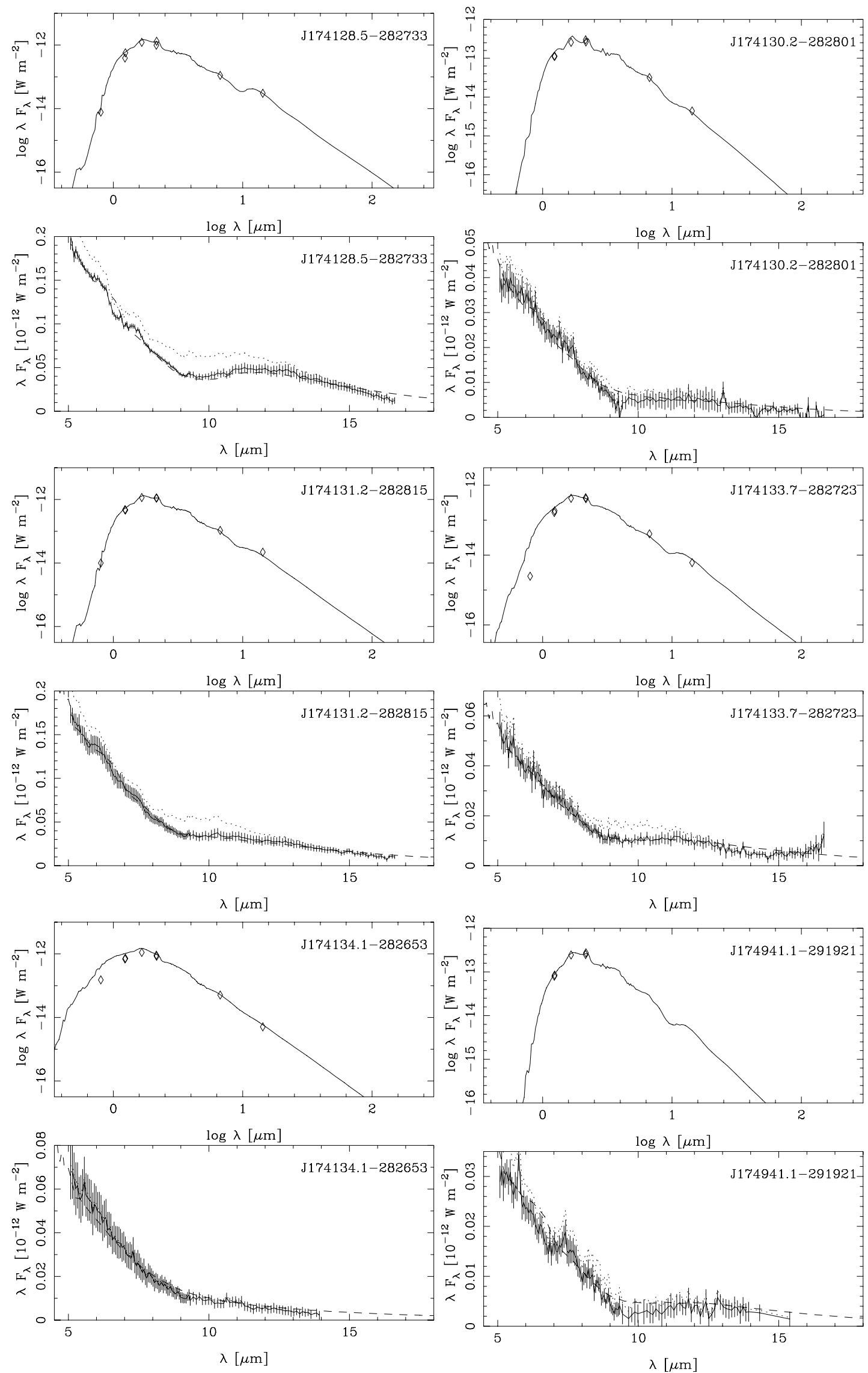

Fig. 2. continued. 
J. A. D. L. Blommaert et al.: ISO Mid-IR spectroscopy of Galactic Bulge AGB stars, Online Material p 4
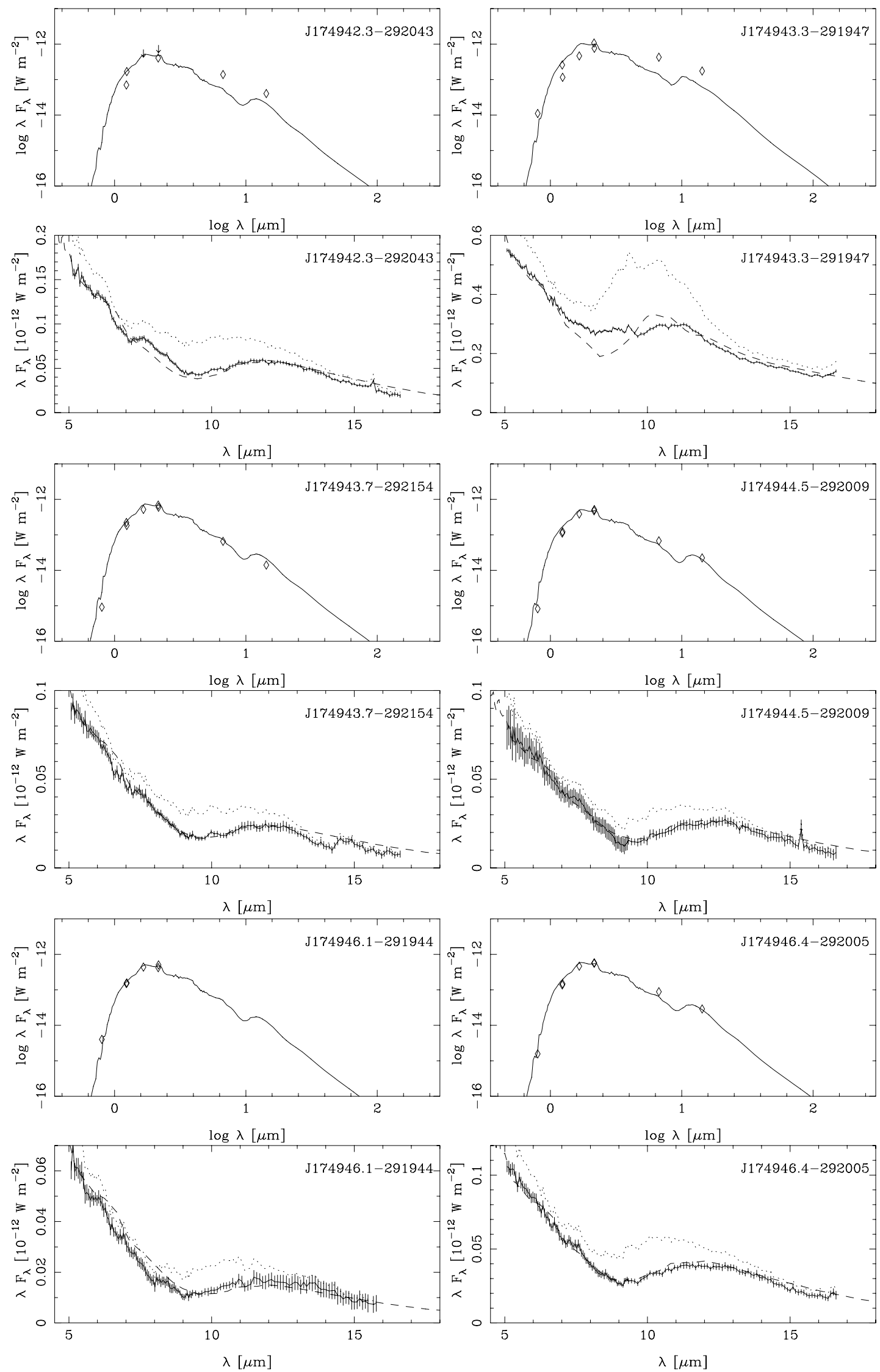

Fig. 2. continued. 
J. A. D. L. Blommaert et al.: ISO Mid-IR spectroscopy of Galactic Bulge AGB stars, Online Material p 5
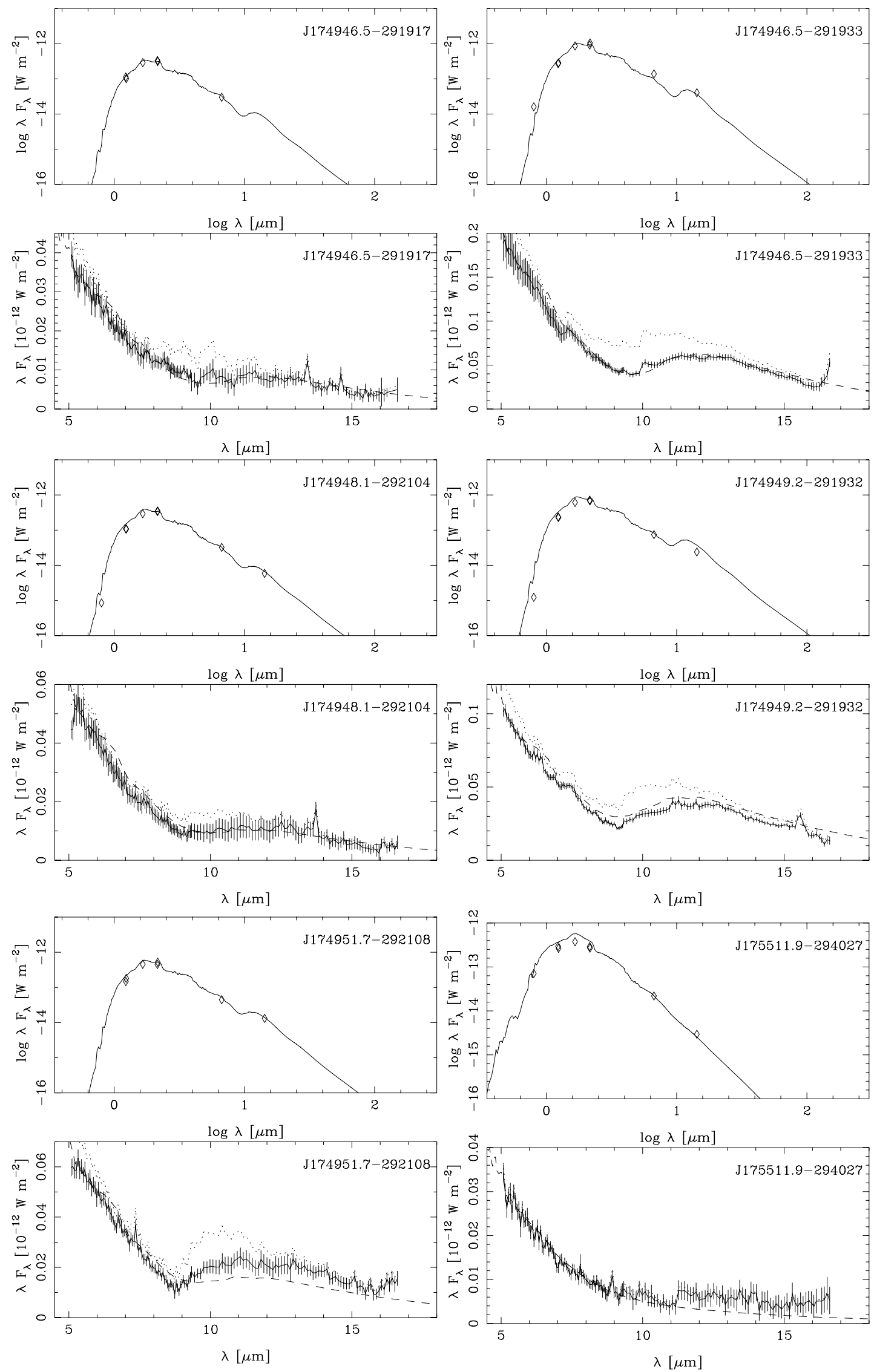

Fig. 2. continued. 
J. A. D. L. Blommaert et al.: ISO Mid-IR spectroscopy of Galactic Bulge AGB stars, Online Material p 6
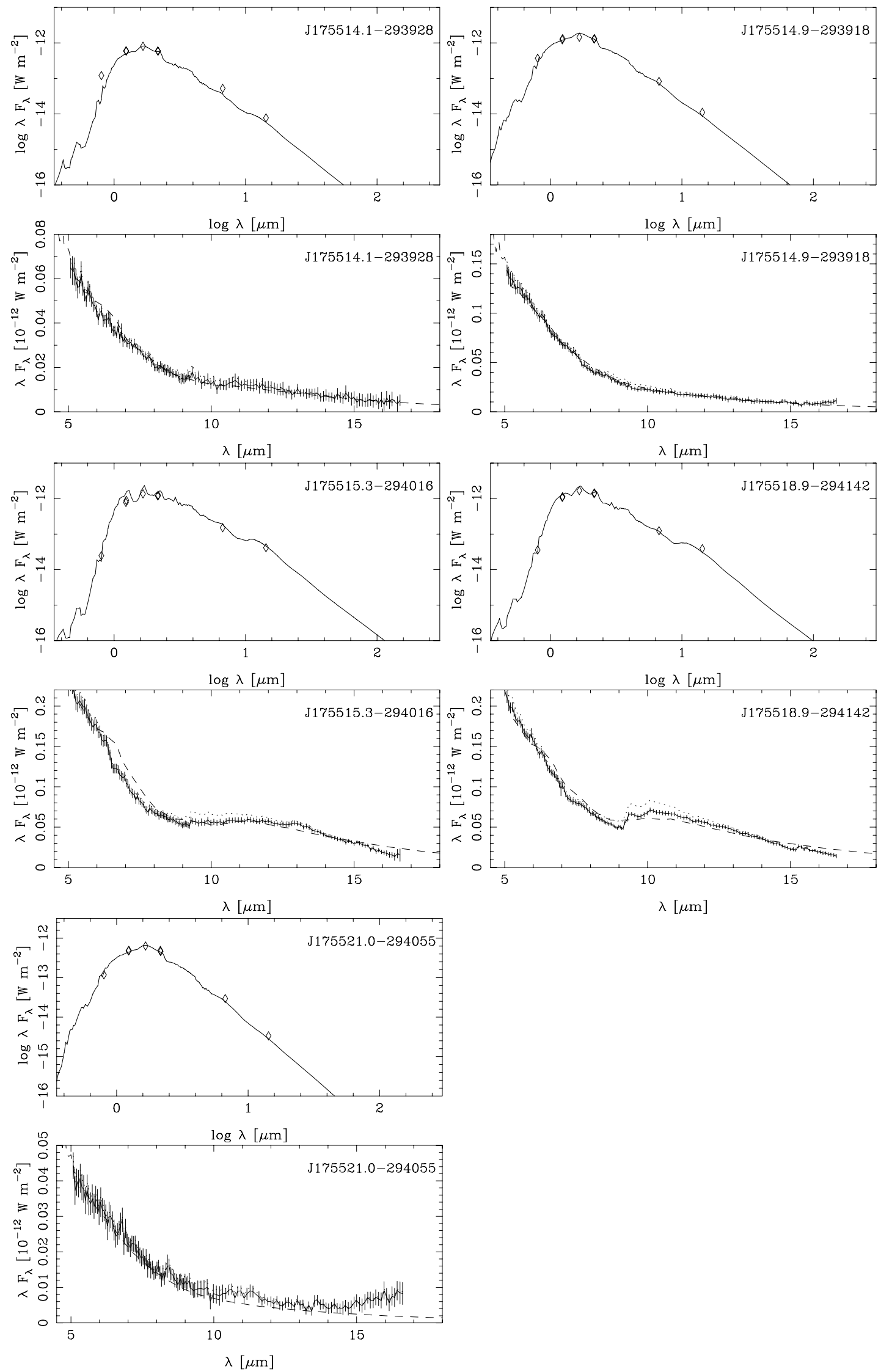

Fig. 2. continued. 